\title{
Independence number of graphs with a prescribed number of cliques
}

\author{
Tom Bohman* \\ Department of Mathematical Sciences \\ Carnegie Mellon University \\ Pittsburgh, PA 15213, U.S.A. \\ tbohman@math.cmu .edu \\ Dhruv Mubayi ${ }^{\dagger}$ \\ Department of Mathematics, Statistics, and Computer Science \\ University of Illinois \\ Chicago IL 60607, U.S.A. \\ mubayi@uic. edu
}

Submitted: Jan 11, 2018; Accepted: Mar 29, 2019; Published: May 17, 2019

(C) The authors. Released under the CC BY-ND license (International 4.0).

\begin{abstract}
We consider the following problem posed by Erdős in 1962. Suppose that $G$ is an $n$-vertex graph where the number of $s$-cliques in $G$ is $t$. How small can the independence number of $G$ be? Our main result suggests that for fixed $s$, the smallest possible independence number undergoes a transition at $t=n^{s / 2+o(1)}$.

In the case of triangles $(s=3)$ our method yields the following result which is sharp apart from constant factors and generalizes basic results in Ramsey theory: there exists $c>0$ such that every $n$-vertex graph with $t$ triangles has independence number at least

$$
c \cdot \min \left\{\sqrt{n \log n}, \frac{n}{t^{1 / 3}}\left(\log \frac{n}{t^{1 / 3}}\right)^{2 / 3}\right\} .
$$
\end{abstract}

Mathematics Subject Classifications: 05C35, 05D10, 05D40

\section{Introduction}

An old problem in extremal graph theory due to Erdős [6] is to determine $f(n, s, l)$, the minimum number of $s$-cliques over all graphs on $n$ vertices and independence number less

*Supported by NSF grant DMS-1362785.

${ }^{\dagger}$ Supported by NSF grant DMS-1300138. 
than $l$. This problem has been extensively studied and has led to many other interesting questions. However, the range of parameters that have been investigated are confined to the case of fixed $l$ (see, e.g., $[5,11,10]$ ).

Here we consider the problem when $s$ is fixed but $l$ can be a power of $n$. It is more convenient to phrase the problem in inverse form as follows. Suppose that $s \geqslant 2$ is fixed and $G$ is an $n$-vertex graph in which the number of copies of $K_{s}$ is $t$, where $0 \leqslant t \leqslant\left(\begin{array}{l}n \\ s\end{array}\right)$. How small can the independence number of $G$ be?

When $s=2$ this question is answered by Turán's theorem. However, when $s \geqslant 3$, one cannot hope to obtain a precise answer for this minimum for the full range of $t$, since when $t=0$ it is equivalent to determining the Ramsey numbers $r(s, l)$ for fixed $s$ and large $l$. Indeed, our motivation for studying this parameter was to gain a better understanding of the Ramsey number $r(4, l)$. In trying to speculate about the growth rate of $r(4, l)$ a natural problem that arises is to determine the smallest possible independence number of a graph where we have a specified number of triangles (the case $s=3$ of our problem).

Our first result below suggests that the behavior of this function changes at $t=$ $n^{s / 2+o(1)}$.

Theorem 1. Let $s \geqslant 2$ be a fixed constant. If $G$ is a graph on $n$ vertices with $t$ copies of $K_{s}$ then

$$
\alpha(G) \geqslant \begin{cases}c_{s} n^{\frac{1}{s-1}} & \text { if } t \leqslant n^{s / 2}, \\ c_{s}\left(\frac{n^{s}}{t}\right)^{\frac{1}{(s)}} \frac{\text { if } t \geqslant n^{s / 2}}{}\end{cases}
$$

\section{Remarks.}

1. The $s=2$ case of Theorem 1 is (apart from constants) simply the bound given by Turán's Theorem.

2. The bound in the case of large $t$ is essentially sharp from random considerations. Indeed, if $G$ is approximately random and has $t$ copies of $K_{s}$ (where $t$ is sufficiently large) then $G$ is roughly $G_{n, p}$ with $n^{s} p^{\left(\begin{array}{c}s \\ 2\end{array}\right)}=\Theta(t)$. Then we expect the independence number of $G$, up to a logarithmic factor, to be $1 / p$. This is exactly the lower bound that we give in the large $t$ case.

3. Although our proof gives some additional log factors, we have chosen to omit those for clarity of presentation. For $s=3$ we carry out the calculations in our method in further detail and provide more precise results below.

When $s=3$ Theorem 1 becomes

$$
\alpha(G) \geqslant \begin{cases}c n^{\frac{1}{2}} & \text { if } t \leqslant n^{3 / 2} \\ c \frac{n}{t^{1 / 3}} & \text { if } t \geqslant n^{3 / 2}\end{cases}
$$

Here the bounds are sharp (apart from logarithmic factors) in the entire range of $t$ due to the existence of Ramsey graphs with the desired independence number $[2,3,7,9]$ 
for $t<n^{3 / 2}$ and approximately random graphs for $t>n^{3 / 2}$. However, our next result sharpens both upper and lower bounds considerably to obtain sharp results in order of magnitude.

Theorem 2. There exists $c>0$ and $n_{0}$ such that if $G$ is a graph on $n>n_{0}$ vertices with $t$ triangles then

$$
\alpha(G) \geqslant \begin{cases}c \sqrt{n \log n} & \text { if } t \leqslant n^{3 / 2} \sqrt{\log n} \\ c \frac{n}{t^{1 / 3}}\left(\log \frac{n}{t^{1 / 3}}\right)^{2 / 3} & \text { if } t \geqslant n^{3 / 2} \sqrt{\log n} .\end{cases}
$$

Both bounds are sharp apart from the constant $c$.

The related question of bounding the chromatic number of a graph with a fixed number of triangles was recently addressed by Harris [8]. We note in passing that the two problems have different characters when the number of triangles is large. The extremal graph for the chromatic number question in this regime is simply a clique while the extremal graph for the independence number question is a direct product of a clique and a Ramsey graph. Harris' proof from [8] uses a difficult theorem of Johansson as its main tool and in fact his bound on chromatic number together with a simple argument can be used to give another proof of Theorem 2. Our proof uses only the lower bounds on independence number of triangle-free graphs; these are much easier results than the corresponding upper bounds on chromatic number.

\section{Proof of Theorem 1}

In the proof, we carry the constant $c_{s}$ through the calculation in the interest of clarity. All conditions that the constants must satisfy are listed as numbered equations below. We make no attempt to optimize these constants.

We go by induction on $s$. Let $d$ be the average degree of $G$. For an arbitrary vertex $x$ let $d(x)$ denote the degree of $x$ and let $t(x)$ denote the number of copies of $K_{s}$ that contain $x$.

Case 1. $t \leqslant n^{s / 2}$.

Here we show that $\alpha(G)>c_{s}^{\prime} n^{\frac{1}{s-1}}$ where $c_{s}^{\prime}$ is a constant (which will be slightly larger than $c_{s}$ itself).

If $d \leqslant\left(c_{s}^{\prime}\right)^{-1} n^{\frac{s-2}{s-1}}$ then we have the desired bound by Turán's Theorem:

$$
\alpha(G) \geqslant \frac{n}{d} \geqslant c_{s}^{\prime} \frac{n}{n^{\frac{s-2}{s-1}}}=c_{s}^{\prime} n^{\frac{1}{s-1}} .
$$

So, we may assume $d>\left(c_{s}^{\prime}\right)^{-1} n^{\frac{s-2}{s-1}}$. Now consider the random variable

$$
X_{v}=d(v)-t(v)^{\frac{2}{s-1}}
$$

where $v$ is a vertex chosen uniformly at random. We have

$$
\begin{aligned}
\mathbb{E}\left[X_{v}\right]=\mathbb{E}\left[d(v)-t(v)^{\frac{2}{s-1}}\right] \geqslant & \left(c_{s}^{\prime}\right)^{-1} n^{\frac{s-2}{s-1}}-\mathbb{E}[t(v)]^{\frac{2}{s-1}} \\
& \geqslant\left(c_{s}^{\prime}\right)^{-1} n^{\frac{s-2}{s-1}}-\left(s n^{\frac{s}{2}-1}\right)^{\frac{2}{s-1}}=\left(\left(c_{s}^{\prime}\right)^{-1}-s^{\frac{2}{s-1}}\right) n^{\frac{s-2}{s-1}}>0 .
\end{aligned}
$$


Note that we assume

$$
c_{s}^{\prime}<s^{-\frac{2}{s-1}} .
$$

Now we apply the inductive hypothesis in $G[N(v)]$ where $v$ is a vertex that achieves the above bound on $X_{v}$. This graph has

$$
d(v)>\left(\left(c_{s}^{\prime}\right)^{-1}-s^{\frac{2}{s-1}}\right) n^{\frac{s-2}{s-1}}
$$

vertices and at most

$$
t(v)<d(v)^{\frac{s-1}{2}}
$$

copies of $K_{s-1}$. Therefore

$$
\alpha(G) \geqslant \alpha(G[N(v)]) \geqslant c_{s-1} d(v)^{\frac{1}{s-2}}>c_{s-1}\left(\left(c_{s}^{\prime}\right)^{-1}-s^{\frac{2}{s-1}}\right)^{\frac{1}{s-2}} n^{\frac{1}{s-1}} .
$$

This gives the desired bound so long as

$$
c_{s}^{\prime} \leqslant c_{s-1}\left(\left(c_{s}^{\prime}\right)^{-1}-s^{\frac{2}{s-1}}\right)^{\frac{1}{s-2}} .
$$

Case 2. $t>n^{s / 2}$.

Here we randomly sparsify our graph and apply Case 1 . We may assume $t<\delta n^{s}$ where $\delta>0$ is a function of the constant $c_{s}$ in the statement of the Theorem.

Set

$$
p=\frac{n}{t^{2 / s} 2^{1+2 / s}}
$$

and consider a set $S$ of vertices chosen at random so that each vertex appears independently with probability $p$. By standard concentration results (using the upper $t<\delta n^{s}$ ) we have

$$
|S|>\frac{n p}{2}=\frac{n^{2}}{2^{2+2 / s} t^{2 / s}}
$$

with high probability. Let $T$ be the number of copies of $K_{s}$ in $G[S]$. By Markov's inequality, we have

$$
T \leqslant 2 E[T]=2 t p^{s}=\frac{n^{s}}{2^{s+1} t}
$$

with probability at least $1 / 2$. Thus, there exists a vertex set $S$ that satisfies both of these inequalities. So we can apply Case 1 to conclude

$$
\alpha(G) \geqslant \alpha(G[S]) \geqslant c_{s}^{\prime}|S|^{\frac{1}{s-1}} \geqslant c_{s}^{\prime} \frac{n^{\frac{2}{s-1}}}{2^{\frac{2(s+1)}{s(s-1)}} t^{\frac{2}{s(s-1)}}} .
$$

This gives the desired bound so long as

$$
c_{s}<c_{s}^{\prime} 2^{-\frac{2(s+1)}{s(s-1)}} .
$$




\section{Proof of Theorem 2}

We begin with the lower bounds. The following result will be used. It follows from the original proof of Ajtai-Komlós-Szemerédi [1] (see also Lemma 12.16 in [4]).

Theorem 3. There exists an absolute constant c such that the following holds. Let $G$ be an $n$-vertex graph with average degree $d$ and $t$ triangles. Then

$$
\alpha(G)>\frac{c n}{d}\left(\log d-\frac{1}{2} \log \left(\frac{t}{n}\right)\right) .
$$

Now we continue with the lower bound proof of Theorem 2 .

Case 1. $t \leqslant n^{3 / 2}(\log n)^{1 / 2}$.

Let $\epsilon=1 / 10$. If $d \leqslant n^{1 / 4+\epsilon}$, then using Turán's theorem we immediately obtain $\alpha(G) \geqslant$ $n /(d+1)>c \sqrt{n \log n}$ with plenty of room to spare, so assume that $d>n^{1 / 4+\epsilon}$.

Next suppose that $d>7 \sqrt{n \log n}$. Let $v$ be a vertex chosen uniformly at random. Let $d(v)$ be the degree of $v$ and let $t(v)$ be the number of triangles containing $v$. Then the expected value of $d(v)-2 t(v)$ is at least $d-6 t / n \geqslant \sqrt{n \log n}$ and since $\alpha(G) \geqslant d(v)-2 t(v)$ we are done. Henceforth we may assume that $n^{1 / 4+\epsilon}<d<7 \sqrt{n \log n}$. Now we apply Theorem 3 to obtain

$$
\alpha(G) \geqslant \frac{c^{*} n}{d} \log \left(\frac{d \sqrt{n}}{\sqrt{t}}\right)>c^{\prime} \sqrt{n \log n}
$$

where $c^{\prime}>0$.

Case 2. $t>n^{3 / 2}(\log n)^{1 / 2}$.

Note that we can assume $t<\delta n^{3}$ where $\delta>0$ is a function of the constant $c$ in the statement of the Theorem. To be precise, $\delta$ can be taken to be solution of the equation

$$
\frac{c}{\delta^{1 / 3}}\left(\log \frac{1}{\delta^{1 / 3}}\right)^{2 / 3}=1 .
$$

Set

$$
p=\frac{1}{4}\left(\frac{n}{t^{2 / 3}}\right)\left(\log \frac{n}{t^{1 / 3}}\right)^{1 / 3}<1 .
$$

Choose a vertex subset $S$ of $V(G)$ by picking each vertex independently with probability $p$. Then, by standard large deviation estimates for $n p$ large and standard Poisson approximation estimates for $n p$ constant, with high probability

$$
|S| \geqslant p n / 2:=N \text {. }
$$

And by Markov's inequality, with probability at least $1 / 2$, the number of triangles $T$ in $S$ satisfies

$$
T<2 p^{3} t=\frac{1}{32}\left(\frac{n^{3}}{t}\right) \log \left(n / t^{1 / 3}\right)<N^{3 / 2} \sqrt{\log N} .
$$


Thus, we can choose a set $S$ that satisfies both of these inequalities and apply the result we have already proved in Case 1 to obtain the lower bound

$$
\begin{aligned}
\alpha(G) \geqslant \alpha(G[S]) \geqslant c^{\prime} \sqrt{N \log N} \geqslant c^{\prime} \sqrt{\frac{n^{2}\left(\log \left(n / t^{1 / 3}\right)\right)^{1 / 3}\left(2 \log \left(n / t^{1 / 3}\right)\right)}{8 t^{2 / 3}}} & \\
& =\frac{c^{\prime}}{2}\left(\frac{n}{t^{1 / 3}}\right)\left(\log \frac{n}{t^{1 / 3}}\right)^{2 / 3} .
\end{aligned}
$$

Note that the last inequality holds for $\delta$, and hence $c$ sufficiently small.

Now we will exhibit constructions showing that these bounds are sharp. In the range $t \leqslant n^{3 / 2}(\log n)^{1 / 2}$ we proceed as follows. Choose $a$ so that $\left(\begin{array}{l}a \\ 3\end{array}\right)=t$ and let $G^{\prime}$ be a trianglefree graph on $n-a$ vertices with independence number at most $c \sqrt{(n-a) \log (n-a)}$ (this exists due to [9]). Note that the upper bound on $t$ implies that $a<2 \sqrt{n} \log n=o(n)$. Let $G$ be the graph that is a disjoint union of $G^{\prime}$ and $K_{a}$. Then $G$ has $n$ vertices, $t$ triangles, and $\alpha(G)=\alpha\left(G^{\prime}\right)+1$.

Next we consider the range $t \geqslant n^{3 / 2}(\log n)^{1 / 2}$. We may assume $t<\delta n^{3}$ for some $\delta>0$. Define $\lambda>0$ by the equation

$$
t=n^{3 / 2} \lambda^{3 / 2} \sqrt{\log \frac{n}{t^{1 / 3}}}
$$

and assume for simplicity of notation that $\lambda$ is an integer. Let $N=n / \lambda$ and again assume that $N$ is an integer. Let $G^{\prime}$ be a triangle-free graph on $N$ vertices and independence number at most $c \sqrt{N \log N}$. Note that $G^{\prime}$ has $\Theta\left(N^{3 / 2} \sqrt{\log N}\right)$ edges. Let $G$ be the lexicographic product of $G^{\prime}$ with $K_{\lambda}$. In other words, replace each vertex $v$ of $G^{\prime}$ with a clique $S_{v}$ of size $\lambda$ and for $x \in S_{v}$, we have $N_{G}(x)=\left(S_{v} \backslash\{v\}\right) \cup \cup_{w \in N_{G^{\prime}}(v)} S_{w}$. Then $G$ has $\lambda N=n$ vertices and the number of triangles in $G$ is $t^{\prime}=\Theta\left(\lambda^{3}\left|E\left(G^{\prime}\right)\right|\right)=\Theta(t)$. Finally,

$$
\alpha(G)=\alpha\left(G^{\prime}\right) \leqslant c \sqrt{N \log N}<c^{\prime} \frac{n}{t^{1 / 3}}\left(\log \left(n / t^{1 / 3}\right)\right)^{2 / 3} .
$$

We have produced a construction where the number of triangles is $t^{\prime}=\Theta(t)$ and it is an easy matter to produce one where the number of triangles is exactly $t$ and the independence number has the same order of magnitude.

\section{Acknowledgments}

The authors thank David Harris for pointing out that the results in [8] can be adapted to yield another proof of Theorem 2. The The first author thanks Gregory and Nina Goossens.

\section{References}

[1] M. Ajtai, J. Komlós, and E. Szemerédi. A note on Ramsey numbers. J. Combin. Theory Ser. A, 29(3): 1980 354-360. 
[2] T. Bohman, The triangle-free process, Adv. Math. 221 (2009) 1653-1677.

[3] T. Bohman and P. Keevash, Dynamic concentration of the triangle-free process. arXiv: 1302.5963

[4] B. Bollobás. Random graphs, volume 73 of Cambridge Studies in Advanced Mathematics. Cambridge University Press, Cambridge, second edition, 2001.

[5] S. Das, H. Huang, J. Ma, H. Naves, B. Sudakov, A problem of Erdős on the minimum number of k-cliques. (English summary) J. Combin. Theory Ser. B 103 (2013), no. 3, 344-373.

[6] P. Erdős, On the number of complete subgraphs contained in certain graphs, Magyar Tud. Akad. Mat. Kutató Int. Közl. 71962 459-464.

[7] G. Fiz Pontiveros, S. Griffiths and R. Morris, The triangle-free process and $R(3, k)$ Mem. Amer. Math. Soc., to appear

[8] D. Harris, Some results on chromatic number as a function of triangle count. arXiv: 1604.00438

[9] J.H. Kim, The Ramsey number $R(3, t)$ has order of magnitude $t^{2} / \log t$, Random Structures Algorithms 7 (1995), 173-207.

[10] V. Nikiforov, On the minimum number of $k$-cliques in graphs with restricted independence number. Combin. Probab. Comput. 10 (2001), no. 4, 361-366.

[11] O. Pikhurko, E. Vaughan, Minimum number of $k$-cliques in graphs with bounded independence number, Combin. Probab. Comput. 22 (2013), no. 6, 910-934. 